$\left(\begin{array}{llr}\text { Jpn. J. Hosp. Pharm. } & \text { P Ph } \\ \hline \text { 䑳 } & \text { 文 } \\ 25(4) & 361-367 & (1999)\end{array}\right)$

\title{
Evaluation of an Automatic Device to Prepare Fibronectin Eyedrops Using a Patient's Own Plasma
}

\author{
HISAHIRO YOSHIDA, KAORI TSUBONE, RIE YAMASHITA, YASUAKI OHTSUBO, \\ TADASHI ISHIMITSU and AKIRA KAMIYA \\ Department of Pharmacy, Yamaguchi University Hospital $\dagger$
}

$\left(\begin{array}{l}\text { Received December 18, } 1998 \\ \text { Accepted March 15, } 1999\end{array}\right)$

\begin{abstract}
Fibronectin (FN) has been reported to be effective for the treatment of corneal trophic ulcers, but the FN eyedrop preparation is not a simple technique. We previously developed an automatic device to prepare FN eyedrops using a patient's own plasma (Yoshida et al. Jpn. J. Hosp. Pharm., 24, 493-498 (1998)). The aim of this study was to evaluate the efficiency of this automatic device. FN eyedrops were prepared using plasma from healthy individuals either with an automatic device or a manual process using gelatin-coupled Sepharose 4 B (GS 4 B) and Sephadex G-25 (PD-10). The average FN concentration and the recovery rate in the eyedrops prepared using the automatic device were $653 \pm 57 \mu \mathrm{g}$ per $\mathrm{ml}$ and $55 \pm 5 \%$, respectively, but those prepared using the manual process were $563 \pm 50 \mu \mathrm{g}$ per $\mathrm{ml}$ and $46 \pm 6 \%$, respectively. The operation of the device was easy and the recovery values of FN were similar among all operators. The total preparation time of FN eyedrops was approximately 1 hour. This automatic device was thus found to be useful for preparing FN eyedrops from the patient's own plasma.
\end{abstract}

Key words - fibronectin, eyedrops, automatic device, corneal trophic ulcer, autologous plasma

\section{Introduction}

Fibronectin (FN) is a glycoprotein, with a molecular weight of about $450 \mathrm{~K}$ dalton, that exists in two forms, plasma FN and cellular. FN. The fibronectin molecule has many binding sites for collagen, fibrin, glycosaminoglycans and cells ${ }^{1-4)}$. FN has been proposed to be responsible for wound healing ${ }^{1,5-7)}$.

Nishida et al. ${ }^{8)}$ reported that they applied purified autologous FN eyedrops prepared from patients exhibiting trophic ulcers with persistent epithelial defects, and that the FN eyedrops markedly improved the condition.

FN eyedrops are difficult to prepare and repetitive use of preparation tubes may cause infections of ethanol-resistant pathogens. Therefore, an automatic device and disposable kit to prepare FN eyedrops should be developed. We examined the optimal conditions for the preparation procedure

$†$ 山口県宇部市南小电1-1-1 ; 1-1-1, Minami-Kogushi, Ube, Yamaguchi, 755-8505 Japan 
of FN eyedrops, and developed an automatic device and disposable kit to prepare FN eyedrops using a patient's own plasma ${ }^{9}$. The aim of this study was to evaluate the efficiency of this automatic device.

\section{Materials and Methods}

\section{Materials}

Human plasma from healthy volunteers, excluded by the alanine aminotransferase (ALT) test, was obtained from the Yamaguchi Red Cross Blood Center (Yamaguchi, Japan). The plasma was divided into plastic disposable test tubes (FQ 2100, Eiken Kizai Co., Tokyo, Japan), and then used in the experiments. The assay kits used for fibronectin and urea were Fibronectin (Opsonic Protein) (Boehringer Mannheim Co., Tokyo, Japan), and BUN reagent A (International Reagent Co., Kobe, Japan), respectively. All chemicals used were obtained from commercial sources and were of analytical reagent grade.

\section{Preparation of FN Eyedrops Using a Manual Process}

FN eyedrops were prepared with plasma from healthy subjects according to the Nishida's manual procedures $^{8)}$ using gelatin-coupled Sepharose 4 B (GS 4 B) and Sephadex G-25 (PD-10). Namely, in the first step (affinity chromatography), citrated plasma was applied to the GS 4 B packed column. The columu was washed with phosphate buffered saline (PBS) containing $10 \mathrm{mM}$ sodium citrate until no protein was detected in the eluates. The protein concentration was monitored with an UV absorbance photometer at $280 \mathrm{~nm}$. Then, FN binding to GS $4 \mathrm{~B}$ was eluted by $4 \mathrm{M}$ urea in 0.05 $\mathrm{M}$ Tris- $\mathrm{HCl}$ buffer, $\mathrm{pH}$ 7.5. The fraction containing $\mathrm{FN}$ was collected $(2.5 \mathrm{~mL})$. In the second step (gel chromatography), the prepacked column of PD-10 was washed and equilibrated by PBS. The FN fraction collected from the GS 4 B column $(2.5 \mathrm{~mL})$ was applied on PD-10 and eluted by PBS. The first $2.5 \mathrm{~mL}$ of the eluate was discarded, and the next $3 \mathrm{ml}$ was collected as the urea-free fraction containing FN.

\section{Preparation of FN Eyedrops Using an Automatic Device}

GS 4 B was packed in a disposable kit (EY-01, Exceeds Co., Ltd., Kobe, Japan) (Fig. 1 ). and

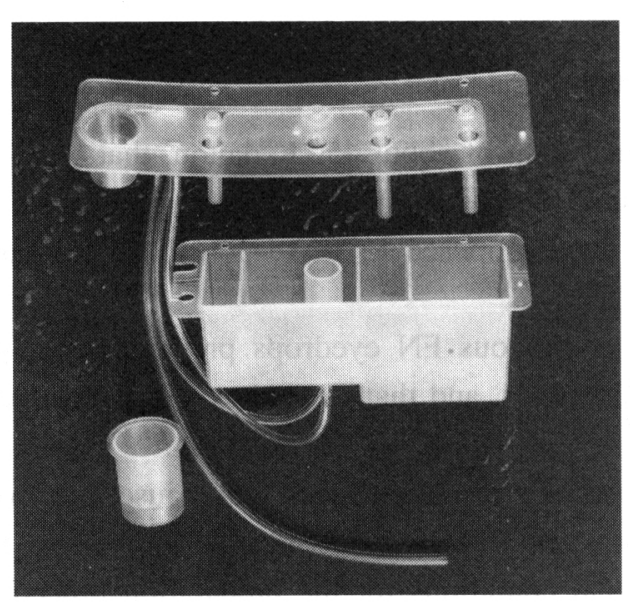

Fig. 1. Disposable Kit for the Automatic Fibronectin Collecting Device (EY-01)

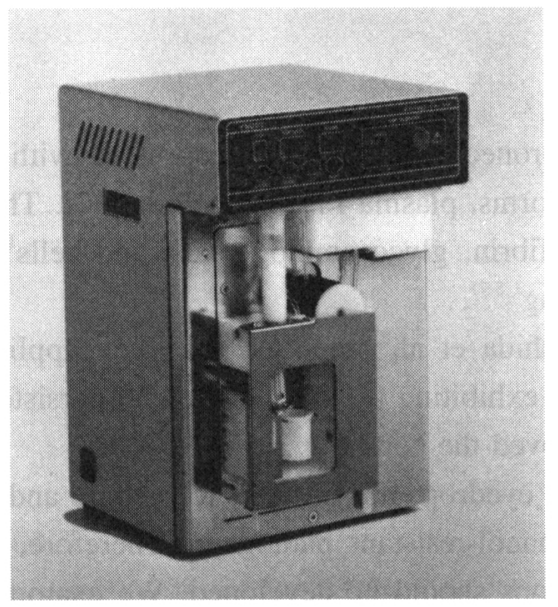

Fig. 2. Automatic Fibronectin Collecting Device (FNCD) 
PBS $(20 \mathrm{~mL})$, citrated PBS $(25 \mathrm{~mL}), 4 \mathrm{M}$ urea $(6 \mathrm{~mL})$, and plasma from healthy subjects $(10 \mathrm{~mL})$ were infused in each section of the kit. This kit was set on an automatic device (FNCD) (Fig. 2) and was equipped with PD-10 and the collecting vessel. Then, the preparation was started. The urea-free fraction containing FN was collected $(3 \mathrm{ml})$.

\section{Determination of $F N$ and Urea}

FN concentrations in citrated plasma from the healthy. volunteers, and those in prepared eyedrops, were determined by the immunoprecipitation method. Urea was assayed by the enzymatic method using urease and glutamate dehydrogenase.

\section{SDS-Polyacrylamide Disc Gel Electrophoresis}

Citrated human plasma, purified human plasma FN with or without prior reduction by 2mercaptoethanol and molecular weight marker proteins (Pharmacia Fine Chemicals) were applied on 4\% SDS-polyacrylamide disc gel, and electrophoresis was performed at room temperature at 5 $\mathrm{mA}$ per tube for 6 hours. Proteins were stained with Coomassie Brilliant Blue G $250(0.01 \%)$ after fixing using $12.0 \%$ trichloracetic acid.

\section{Biological Activity Assay}

To determine the biological activity of FN, we measured the cell adhesion activity ${ }^{10)}$ of purified FN according to the neutral red method ${ }^{11)}$. In brief, baby hamster kidney cells (BHK cells) were cultured and single cell suspensions of BHK cells were placed in a multiwell cultured plate precoated with fibronectin. BHK cells that adhered to the well were incubated in neutral red solution, and the amount of stain accumulated in the cells was monitored by spectrophotometry at $540 \mathrm{~nm}$.

\section{Results And Discussion}

\section{Preparation of FN Eyedrops Using an Automatic Device}

Firstly, examination of the concentration and recovery rate of FN from plasma using an automatic device or manual process was performed. The results are shown in Table 1 . The average FN concentration and the recovery rate in the eyedrops prepared with the device were $653 \mu \mathrm{g}$ per $\mathrm{mL}$ and $55 \%$, respectively, and those prepared with the manual process were $563 \mu \mathrm{g}$ per $\mathrm{mL}$ and $46 \%$, respectively. Variations of the FN recovery rate in the eyedrops with the device were narrower than those with the manual process (Fig. 3 ). These results suggest that our device was more effective to obtain high concentrations and low variations of FN eyedrops than the manual process. Nishida et al. ${ }^{12)}$ reported that the clinical efficacy of FN eyedrops in patients with corneal epithelial lesions was dependent on the FN concentration. Therefore, preparing eyedrops with high FN concentrations is important to obtain good clinical efficacy in patients with corneal epithelial lesions.

Secondly, the total preparation time for FN eyedrops with the automatic device was compared to

Table 1. Effects of the Preparation Method on the Yield of Fibronectin

\begin{tabular}{lcc}
\hline & Automatic device & Manual process \\
\hline FN conc in plasma $(\mu \mathrm{g} / \mathrm{mL})$ & $356 \pm 37$ & $362 \pm 44$ \\
FN conc in eyedrops $(\mu \mathrm{g} / \mathrm{mL})$ & $653 \pm 57$ & $563 \pm 50$ \\
Recovery rate $(\%)$ & $55 \pm 4.9$ & $46 \pm 5.8$ \\
$\mathrm{n}$ & 15 & 20 \\
\hline
\end{tabular}

Mean士S. D. 
that with the manual process. As shown in Table 2, the former method required about $60 \mathrm{~min}$, which was less time than the latter method. Moreover, the operator's handling time with the former method was markedly shorter than that with the latter, and operation of the device was very easy. The main cost to prepare FN eyedrops with the device and the manual process was the cost of the disposable kit and personnel expenditures, respectively. Our automatic device can only be used to prepare FN solutions, but devices used in the manual process (UV monitor, pen recorder and peristaltic pump) are widely used for many purposes. Using the disposable kit may prevent pathogenic

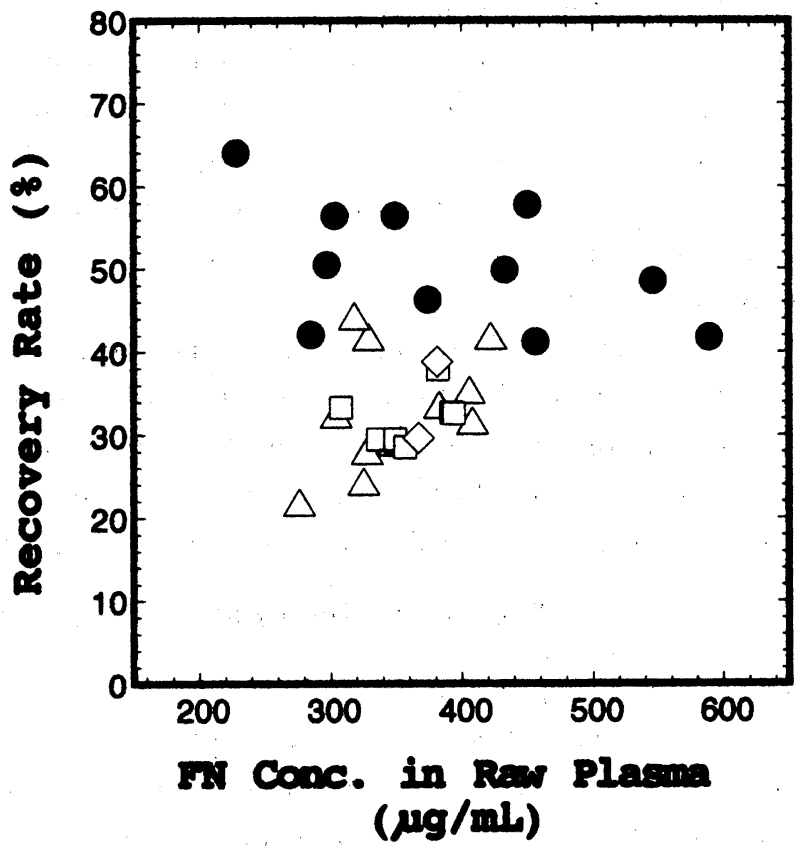

Fig. 3. Relationship between the Fibronectin Concentration in Raw Plasma and the Recovery Rate in Fibronectin Eyedrops

$\checkmark$, FN eyedrops prepared using the automatic device; $\Delta, \diamond$, $\square$, FN eyedrops prepared according to manual procedure by operator 1,2 or 3 .

Table 2. Comparative Study

\begin{tabular}{lcc}
\hline Item & Automatic device & Manual process \\
\hline Total preparation time & $60 \mathrm{~min}$ & $90 \mathrm{~min}$ \\
Handling time & $10 \mathrm{~min}$ & $60 \mathrm{~min}$ \\
Procedure & easy & difficult \\
Infection between patients & no & possible \\
FN concentration in eyedrops & high & normal \\
Recovery rate & high & normal \\
Variation of recovery & narrow & broad \\
Main cost & disposable kit & man power \\
Use of device & specific & various \\
\hline
\end{tabular}


infections because contamination with other samples is avoided. Our automatic device was more effective to obtain high concentrations and low variations of FN eyedrops than the manual process. These results indicate that the device effectively reduced the time and labor required for FN eyedrop preparation, and that FN therapy can be easily applied to outpatients as well as inpatients.

\section{Purity and Activity of FN Eyedrops}

The purity of FN prepared with the device was examined. Fig. 4 shows the electrophoretic pattern of FN eyedrops with or without prior reduction by 2-mercaptoethanol and heat. FN eyedrops prepared with the device mainly contained $450 \mathrm{~K}$ dalton FN and some contained $220 \mathrm{~K}$ dalton FN similar to that prepared by the manual process. When these two forms of FN were reduced, all FN became $220 \mathrm{~K}$ dalton protein. These results clearly show that FN eyedrops Prepared with the device were electrophoretically pure.

The biological activity of FN produced by the device was then examined. The FN prepared with the device had the same potency of the adhesion effect on BHK cells as that with the manual process. The relationship between the biological activity and immunological activity of FN eyedrops is shown in Fig. 5. The biological activity was closely correlated with the immunological activity of FN eyedrops. Thus, the FN prepared with the device may exhibit biological activity, and its activity may be easily predicted by immunological methods.

The stability of the FN eyedrops in immunological activity was examined at room temperature. FN showed the same degree of biological and immunological activity after 7 days at room temperature. Thus, prepared FN eyedrops may be stable for at least 7 days under conventional conditions.
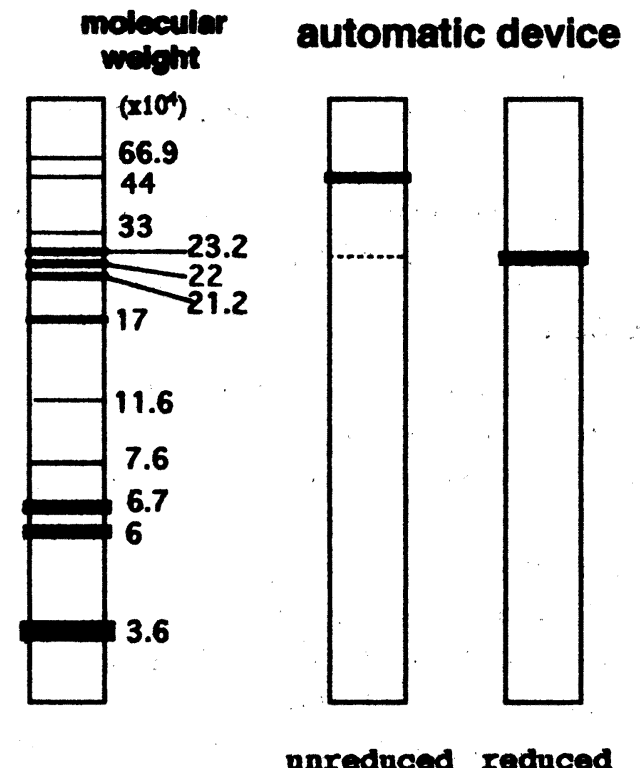

manual process
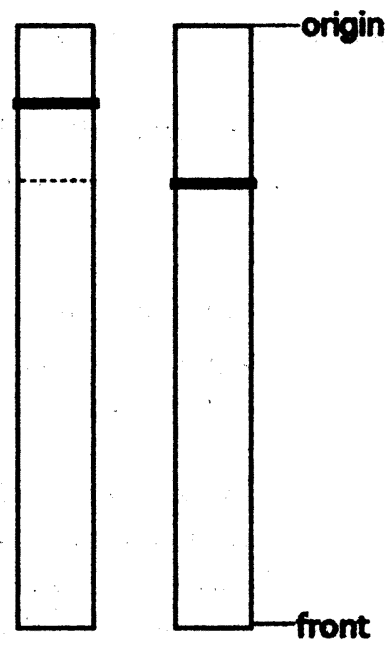

unreduced reduced

Fig. 4. Electrophoretic Pattern of Fibronectin Eyedrops Prepared Using an Automatic Device or Manual Process 


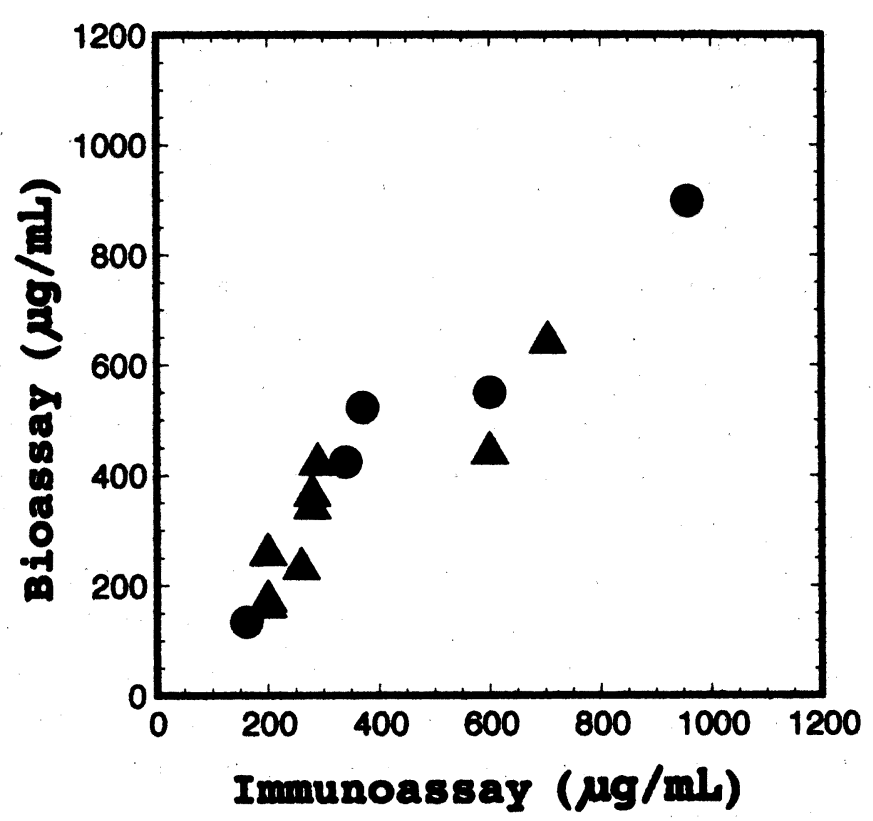

Fig. 5. Relationship between the Biological and Immunological Activity of FN Eyedrops Prepared Using an Automatic Device or Manual Process

O, FN eyedrops prepared using the device $(R=0.954, Y=0.85 X$ $+91) ; \Delta:$ FN eyedrops prepared according to manual procedure $(R=0.879, Y=0.72 X+105)$.

These results indicate that our automatic device is very useful to prepare FN eyedrops from a patient's own plasma.

Acknowledgement We gratefully acknowledge the Yamaguchi Red Cross Blood Center for donating human plasma from healthy volunteers excluded by the ALT test.

\section{References}

1) E. Pearlstein, L. I. Gold, A. Garcia-Pardo, Mol. Cell Biochem., 29, 103-126 (1980).

2) E. Ruoslahti, E. Engvall, E. G. Hayman, Coll Res., 1, 95-128 (1981).

3) E. Ruoslahti, E. G. Hayman, E. Engvall, Tumor Cell Surfaces and Malignancy, ed. by Liss A. R., 1980, pp.821-828.

4) K. M. Yamada, K. Olden, Nature, 275, 179-184 (1978).

5) T. Soria, N. Nishida, Y. Ohashi, S. Nakagawa, R. Manabe, Curr, Eye Res., 1, 553-556 (1981).

6) R. Manabe, T. Nishida, Y. Ohashi, S. Nakagawa, T. Awata, H. Tanaka, T. Suda, K. Watanabe, Y. Inoue, C. Nishibayashi, Acta Soc. Ophthalmol. Jap., 88, 401-413 (1984).

7) N. Nishida, S. Nakagawa, T. Awata, Y. Ohashi, K. Watanabe, R. Manabe, J. Cell Biol ., 97, 1653-1657 (1983).

8) T. Nishida, S. Nakagawa, T. Awata, C. Nishibayashi, R. Manabe, Jpn. J. Ophthalmol., 26, 416-424 (1982). 
9) H. Yoshida, R. Yamashita Y. Ohtsubo, T. Ishimitsu, A. Kamiya, Jpn. J. Hosp. Pharm., 24, 493-498 (1998).

10) F. Grinnell, J. Cell Sci. 65, 61-72 (1984).

11) E. Borenfreund, J. A: Puerner, Toxicol. Letters, 24, 119-124 (1985).

12) T. Nishida, J. Yagi, T. Otori, K. Watanabe, R. Manabe, H. Imagama, N. Miyata, Jpn. Clin. Ophthalmol., 42, 33-37 (1988). 